[Agr. Biol. Chem., Vol. 29, No. 10, p. 965 966, 1965]

\title{
New Pterin Produced by Aspergillus
}

Sir:

Previously the author and his collaborators have observed fluorescent products in 249 strains of Aspergillus belonging to Asp. flavus, oryzae, niger, tamarii, japonicus, glaucus, AwamoriKoji, Shochu-Koji and so on, incubating them in Pfeffer's liquid media and irradiating the broths with ultraviolet lamp."

The fluorescent products recognized so far were classified largely into 3 groups, namely (a) yellow fluorescent material, this is riboflavin, (b) markedly bluish purple fluorescent substance, (c) yellowish green fluorescent materials. These are considered to consist of several compounds, to which Aflatoxin ${ }^{2 !}$ might possibly belong, if, as in rare case, the Aspergillus were a producing strain of it; besides, the other one which was isolated as a crystalline form already by the author. ${ }^{3}$ '

In this communication he wishes to deal with this (b) bluish purple fluorescent substance (abbreviated to VS), informing of its production, isolation to a crystalline form and identification as a new pterin.

Asp. oryzae (Ahl.) CoHN was incubated in about $1000 \mathrm{l}$ of modified Pfeffer"s medium containing $100 \mathrm{~g}$ sucrose and $30 \mathrm{~g}$ ammonium nitrate per 1 at $30^{\circ} \mathrm{C}$. By stationary culture in duralumin vessel, carbon source was consumed in about 45 days, organic acid products, such as Kojic acid, reached their max. in 45 50 days, then decreased, and mycelium crop yields became max. in about 50 days. VS was formed proportionally to cell growth and did not decrease after attaining to max. amount of VS.

Paper chromatography ( $n$-butanol-acetic acid-

1) Y. Kaneko, K. Sakaguchi and Y. Kihara, J. Ferm. Tech., 27, 156 (1949).

2) A. Takagi, and K. Aihara, J. Food Hygienic Soci. Japan, 6, 1 (1965).

3) Y. Kaneko and S. Umemoto, Proc. 29 th Meeting Central Branch Agri. Chem. Soci. Japan, (1961) water, $4: 1: 5)$ of VS showed two fluorescent spots at $R_{F} 0.24$ (VS-I) and 0.14 (VS-II).

The broth was filtered after 55 days incubation and the mycelial mat was ground with quartz sand from which the fluorescent materials were extracted with hot water. VS in the filtrate and extract were then adsorbed onto charcoal at $\mathrm{pH} 7.5$. After being eluted from the carbon cake with $0.1 \mathrm{~N}$ caustic sodaacetone mixture $(4: 1)$, the VS eluates were neutralized immediately and concentrated in vacuo. From the concentrate ether-soluble materials such as yellow pigments and yellowish green fluorescent substances were removed by continuous ether extraction at $\mathrm{pH} 3.5$. VS in aqueous layer were adsorbed again onto charcoal and eluted with alkali acetone similarly as before.

The concentrated eluate was treated with 9 times volume of methanol, VS being dissolved into the 90\% methanol layer. The extract was passed through alumina column from which at first the adsorbed VS-I was eluted with $80 \%$ methanol, then VS-II with $60 \%$ methanol. VS-II fraction was purified once more by similar alumina chromatography.

Dowex-I, X-4, $-\mathrm{CO}_{3}$ form colum chromatography was thereafter applied to VS-II solution which was adsorbed on the top of the column and eluted by hydrochloric acid with batchwise exchange. Further purification was fulfilled by Filtrol Grade 58 (Adsorbent made by Filtrol Corporation U.S.A.) column chromatography, VS-II having been adsorbed at $\mathrm{pH} 3.0$ and eluted with water containing increasing amount ( $1 \%, 2 \%$ and so on) of acetone.

VS-II was thus completely separated from minute VS-I contaminant and finally treated with Dowex-I column chromatography again. The eluate was concentrated to yield pale 
yellow needles of VS-II of about $400 \mathrm{mg}$.

Recrystallization from hot water afforded fine needles of m.p. $193^{\circ} \mathrm{C}$ (with decomp.), $[\alpha]_{D}^{20}-60.2^{\circ} \quad\left(c=3.321 \times 10^{-3} \mathrm{~g} / \mathrm{ml}, \quad \mathrm{l}=1 \mathrm{dm}\right)$. Potentiometric titration of the compound demonstrated pKa 8.0 and m.w. 358.9 .

Anal. Found: C, 42.16; H, 5.19; N, 18.23 Calcd. for $\mathrm{C}_{13} \mathrm{H}_{13} \mathrm{O}_{7} \mathrm{~N}_{5} \cdot \mathrm{H}_{2} \mathrm{O}: \mathrm{C}, 41.82 ; \mathrm{H}, 5.13$; $\mathrm{N}, 18.76 \%$ m.w. 373.3 .

The compound is considerably soluble in water, phenol, moderately soluble in methanol, pyridine, acetic acid, sparingly soluble in ethanol, $n$-butanol, acetone, benzyl alcohol and insoluble in ether, ethylacetate, chloroform, benzene, petroleum ether. Its solution except in phenol exhibits brilliant bluish purple fluorescence especially in alkaline $\mathrm{pH}$ water.

The ultraviolet absorption spectra in Fig. 1 shows $\lambda_{\max } 259,281,360 \mathrm{~m} \mu$ in $0.1 \mathrm{~N}$ sodium hydroxide and $293,347 \mathrm{~m} \mu$ in $0.1 \mathrm{~N}$ hydrochloric acid. This indicates the fact that VSII belongs to a typical isoxanthopterin derivative though some bathochromic shift exists.

Basing upon its elemental analysis, m.p. and ultraviolet spectra it is quite obvious that VS-II is a new pterin occurring in nature.

The author wishes to express his heartfelt gratitude to Dr. Kinichiro Sakaguchi, Emeritus Professor, Dr. Kei Arima, Professor, the

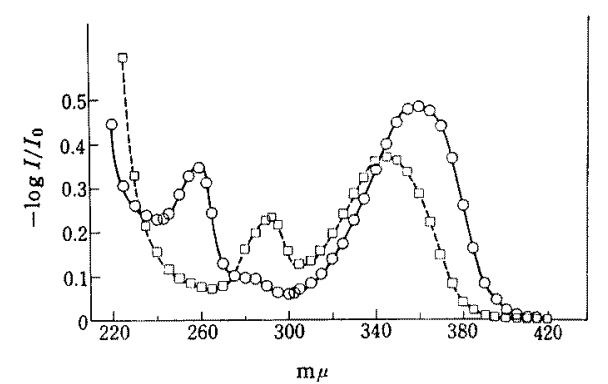

FIG. 1. Ultraviolet Absorption Spectra of VS-II.

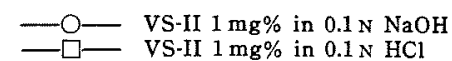

University of Tokyo, and Dr. Shinji Doi, Professor, Nagoya University, for their direction and encouragement through this work. He also wishes to dedicate his thanks to Dr. Tadao Hama and his associates, Keio University, and fellow researchers in Tokyo and Nagoya Universities for their kind help rendered to his study. The author is much indebted to Wakamoto Pharmaceutical Co., Dainippon Sugar Co. and Daito Sugar Co. for their kind assistance to large scale cultivation of the Aspergillus.

Yasuyuki $\mathrm{K}_{\text {ANEKO }}$

Department of Agricultural Chemistry, Nagoya University, Anjo, Aichi-Ken

Received August 19, 1965 